\title{
Cross-Layer Hybrid FEC/ARQ Reliable Multicast with Adaptive Modulation and Coding in Broadband Wireless Networks
}

\author{
Reuven Cohen Guy Grebla Liran Katzir \\ Department of Computer Science \\ Technion-Israel Institute of Technology \\ Haifa 32000, Israel
}

\begin{abstract}
In this paper we define and address a new problem that arises when a base station in a broadband wireless network wishes to multicast information to a large group of nodes and to guarantee some level of reliability using Application layer FEC codes. Every data block to be multicast is translated into a sequence of $K+n$ packets, from which every receiver must receive at least $K$ in order to correctly decode the block. The new problem is to determine which PHY layer MCS (Modulation and Coding Scheme) the base station should use for each packet. We present several variants of this problem, which differ in the number of ARQ (Automatic Repeat reQuest) rounds during which the delivery of a data block must be completed. Most of these variants are shown to be NP-hard. However, we present optimal solutions for practical instances, where the number of MCSs is small, and efficient approximations and heuristics for the general case of each variant.
\end{abstract}

\section{INTRODUCTION}

A prominent feature of advanced wireless technologies such as WiMax/802.16 [10] and 3GPP/LTE [1] is the base station's ability to transmit a single copy of a packet to a group of receivers, a concept known as multicast. Indeed, streaming multicast is considered as one of the most important applications in such networks.

To ensure some level of reliability, streaming multicast often uses Application layer FEC (Forward Error Correction) codes, with or without ARQ (Automatic Repeat reQuest). In a typical FEC-based multicast, the sender creates from each data block $K+n$ packets, and every receiver must receive any $K$ of these packets in order to correctly decode the data block [17]. In rateless erasure codes, the value of $n$ can be different for different data blocks.

Application layer FEC codes can be classified into two main groups: near-optimal codes and optimal codes. In near-optimal codes, $(1+\epsilon) \cdot K$ packets are required in order to correctly decode the data block, while in optimal codes, $K$ packets are required. Throughout this paper we assume that an MDS (Maximum Distance Separable) code is used in the application layer FEC [7], [18]. MDS is a family of optimal codes that includes the well known Reed-Solomon code.

In a hybrid FEC/ARQ-based scheme [3], [8], [16], [19], receivers that have not received enough packets notify the sender by sending a NACK message [2], and the sender may send additional repair packets. The number of such repair rounds is, in practice, limited by real-time, buffer space, and similar considerations.

Adaptive modulation and coding (AMC) is crucial for increasing the performance of broadband wireless networks. With AMC, the base station usually uses higher order modulation (such as 16- or 64-QAM) and higher code rate (such as $\mathrm{R}=3 / 4$ turbo code) when transmitting unicast packets to nearby receivers, and lower order modulation (such as QPSK) and code rate when transmitting unicast packets to distant receivers. Multicast packets, however, are usually transmitted using low order modulation and coding, because of the very high probability that at least one of the receivers is not close enough to the base station.

In this paper, we show that the base station can improve the performance of multicast by optimizing the selection of an MCS for each individual packet. We are not aware of any previous work that has addressed this cross-layer combination of Application layer hybrid FEC/ARQ with physical layer Adaptive Modulation and Coding (AMC). Therefore, to the best of our knowledge, not only are the theoretical results and algorithms presented in this paper new, but so is the problem itself.

The new problem we define is referred to as RM-AMC (Reliable Multicast using Adaptive Modulation and Coding). RM-AMC has two main variants: for a pure FEC scheme, where only one round is used for the delivery of every data block, and for a hybrid FEC/ARQ scheme, where multiple rounds can be used. With one round, the base station sends $K+n$ packets for every data block and must decide:

- what the value of $n$ should be;

- what MCS should be used for each of these $K+n$ packets.

With multiple rounds, the sender needs to address these issues not only for the first round, but for every additional one.

It is important to note that in the considered model FEC is used at the application layer and MCS at the PHY layer. Therefore, the Application layer of the receiver can correctly decode the data block if it receives any $K$ packets, regardless of the MCSs used to transmit these packets in the PHY layer.

The RM-AMC problem defined in this paper and the algorithms for solving it rely heavily on the concept of cross-layer optimization. That is, information retrieved by a lower layer 
(PHY) is used by an upper layer (Application/Transport) in order to improve the performance of the upper layer's protocol.

The rest of this paper is organized as follows. In Section II, we discuss related work. In Section III, we describe the considered multicast service model, define the RM-AMC problem, and prove that it is NP-hard. In Section IV, we present several algorithms for RM-AMC. In Section V, we extend RM-AMC to multiple rounds and present a simulation study of the various algorithms in Section VI. In section VII, we extend our results to more optimization criteria. Finally, Section VIII concludes the paper.

\section{RELATED WORK}

In recent years, the number of important applications for multicast in broadband access wireless networks has been growing steadily. One such application is Internet Protocol Television (IPTV) over Wimax [21], [25], which is supposed to enable mobile users to receive streaming video content.

The concept of reliable multicast for streaming and other applications has been addressed by the IETF RMT (Reliable Multicast Transport) working group. This working group has published several RFCs on large-scale multicast. The main protocol developed by the RMT WG for large-scale reliable streaming multicast is called NORM (NACK oriented reliable multicast) [2], which employs the concept of hybrid FEC/ARQ [8], [16], [19], [23], [22]. For a good overview of the RMT WG, see [1].

In [9], problems related to MAC layer multicast are studied. This paper does not study Application layer hybrid FEC/ARQ for reliable multicast, but is more concerned with Physical layer transmission codes. When the sender wants to send a message, it splits it into several hierarchical layers and transmits each layer using its own MCS (modulation and coding scheme). The MCS depends on the importance of the encoded layer. Similar ideas are also presented in [14].

In [13], three schemes to adaptively change the MCS of multicast packets are discussed. In each scheme, the sender uses the channel conditions of the receivers to determine, for every packet, which MCS to use. The three schemes have different reliability and throughput. However, unlike our work, [13] does not use FEC or ARQ.

In [8], Application layer FEC/ARQ is used, but without AMC. The sender encodes every data block into multiple packets. It is then supposed to get feedback messages from the receivers in order to decide how many more packets to send for the same data block. This is the standard Application layer hybrid FEC/ARQ proposed by NORM. In [20], convolutional coding and nonuniform PSK modulation are combined to provide greater efficiency. Nonuniform PSK is used to transmit additional information to the more capable receivers.

In [24] and [26], the authors introduce and analyze a crosslayer framework for video multicast. Several video layers are generated and $b_{i}$ data blocks, each of $K_{i}$ bytes, are used for every layer $i$. Each data block is encoded and expanded into $N$ bytes using an $\left(N, K_{i}\right)$ Reed-Solomon code. Then, a packet composed of one byte from every data block is generated using a modified multiple description coding scheme (MDC) in which superposition coding is used to encode each layer using a different MCS. In [26], an analysis is performed for the worst receiver in a Rayleigh channel. Neither [24] and [26] consider ARQ. In addition, in both frameworks, every data block is encoded into $N$ bytes, where $N$ is the same for all data blocks.

In [5], a layered coding approach that uses error correction coding within each packet and erasure correction coding across the packets is proposed. The authors consider a Nakagami wireless channel and optimize the transmission assuming the transmission rate is continuous. They show that the performance is close to optimal when the transmission is performed using a set of known MCSs. ARQ and multicast transmission are not considered in this paper.

In [27], optimal partitioning of receivers into groups for multirate multicast is studied. A dynamic programming algorithm that finds an optimal partition is presented. In [11], algorithms for the problem of maximizing the aggregate receiver utility for the case of multirate multicast sessions are presented. As in our work, several MCSs are used in order to increase performance. However, Application layer FEC/ARQ is not applied.

\section{PRELIMINARIES}

\section{A. Reliable multicast streaming service model}

In this paper we consider a streaming multicast service for which full reliability is neither possible nor essential. It is not possible due to: (a) occasionally bad wireless channel conditions and intermittent disconnection introduced by mobility of the hosts; (b) the streaming nature of the broadcast data, which puts hard limits on the time the delivery of every data block must be completed. Full reliability of streaming multicast is not essential because streaming applications (audio and video) can tolerate data loss. If the loss is temporary, it might not even be noticed by the user due to the robustness of the audio/video codecs. If the loss is long in duration, e.g., due to a physical obstacle between a mobile node and the base station, the user will probably want to continue receiving the audio/video multicast despite the blackout period.

For the RM-AMC problem defined in this paper, one may consider several optimization criteria, all of which are related to the "designated group." This group does not include all the nodes that join the multicast group, but only those whose wireless channel is "not too bad" because satisfying nodes whose wireless channel is too bad would consume too much bandwidth. The designated group contains only nodes whose SNR is above some threshold. Those are the nodes to which some level of QoS has to be guaranteed. The optimization criterion considered throughout most of this paper is:

OC-1 Let $p_{i}$ be the probability that the $i$ th receiver of the designated group will correctly decode the data block. Maximize $\min _{i}\left(p_{i}\right)$, while guaranteeing that the total bandwidth is not larger than $B_{\max }$.

In Section VII, we address other optimization criteria as well.

Consider a multicast packet sent by the base station. The probability that a certain receiver will correctly receive this packet is determined by the receiver's SNR (signal-to-noise 
ratio). Throughout the paper we assume that for two receivers $a$ and $b$, if the SNR of $a$ is higher than the SNR of $b$, then the probability that $a$ will correctly receive a multicast packet is not smaller than the probability that $b$ will correctly receive the same packet. This is true regardless of the MCS used by the base station for the PHY layer encoding of this packet. This implies that in OC-1, the minimum probability should only be guaranteed to the receiver with the worst SNR from the designated group. For the rest of the paper, such a receiver will be referred to as the designated receiver.

\section{B. Using one or more rounds}

An optimal solution for RM-AMC $(\mathrm{OC}-1)$ depends on the number of rounds the sender can use for sending the packets of a certain data block. If only one round is possible, the sender needs to decide how many packets should be sent in this round and what MCS should be used for each of them. These packets are then transmitted, and no more packets can be used for this data block.

If $R>1$ rounds are possible, we assume that after every round of transmission the sender receives a feedback message about the outcome of the previous round. The sender will use this information to decide how many new packets should be broadcast in the next round for the same data block, and what MCS should be used for each.

The exact feedback the base station should receive in every round depends on the optimization criteria we want to address. Receiving a feedback message from every individual receiver is impractical because it leads to the well-known feedback implosion problem. For OC-1 it is sufficient to receive a feedback message from only one receiver, as discussed in Section V-A.

\section{The effect of AMC on schedule efficiency}

In what follows, we give some examples of the relationship between the PHY layer AMC and the schedule efficiency. Consider two MCSs, MCS-1 and MCS-2. Suppose that when a packet is encoded using MCS-1, it requires twice the bandwidth required by MCS-2. On the other hand, suppose that the probability that the designated receiver will correctly receive an MCS-1 packet is $1-\epsilon$, where $\epsilon$ is very close to 0 , and the probability that it will correctly receive an MCS-2 packet is only $\frac{1}{2}$. Suppose also that $K=2$ and that the bandwidth $B$ is sufficient for (a) 2 MCS-1 packets, or (b) 4 MCS-2 packets, or (c) 1 MCS-1 packet and 2 MCS-2 packets. With only one round, the best choice is (a). Using this option, the probability that the designated receiver will correctly decode the data block is $(1-\epsilon)^{2}$, compared to $\left(\begin{array}{l}4 \\ 2\end{array}\right)\left(\frac{1}{2}\right)^{4}+\left(\begin{array}{l}4 \\ 3\end{array}\right)\left(\frac{1}{2}\right)^{4}+\left(\begin{array}{l}4 \\ 4\end{array}\right)\left(\frac{1}{2}\right)^{4}=\frac{11}{16}$ for option (b), or $2(1-\epsilon) \cdot \frac{1}{2} \cdot \frac{1}{2}+\epsilon \cdot \frac{1}{2} \cdot \frac{1}{2}+(1-\epsilon) \cdot \frac{1}{2} \cdot \frac{1}{2} \approx \frac{3}{4}-\epsilon$ for option (c).

If $K=2$ and the available bandwidth $B$ is sufficient for transmitting only 1 MCS-1 packet or 2 MCS- 2 packets, the best choice is of course the latter, because the success probability is $\frac{1}{4}$ compared to 0 .

Finally, suppose that the available bandwidth $B$ is sufficient for transmitting 3 MCS-2 packets or 1 MCS-1 packet and 1
MCS-2 packet. In this case, the best choice is to transmit 3 MCS-2 packets. The probability that the designated receiver will correctly decode the data block is $\left(\begin{array}{l}3 \\ 2\end{array}\right) \cdot \frac{1}{2}^{3}+\left(\begin{array}{l}3 \\ 3\end{array}\right) \cdot \frac{1}{2}^{3}=\frac{1}{2}$, compared to $(1-\epsilon) \cdot \frac{1}{2}=\frac{1}{2}-\frac{\epsilon}{2}$ using only 1 MCS-1 packet and 1 MCS-2 packet.

\section{Combining multiple rounds and multiple MCSs}

To see how we can increase the performance by increasing the number of rounds, suppose that $K=2$ and that the available bandwidth $B$ is sufficient for transmitting 3 MCS2 packets or 1.5 MCS-1 packets. Suppose also that the probability that the designated receiver will correctly receive an MCS-1 packet is $1-\epsilon$, and the probability that it will correctly receive an MCS-2 packet is $\frac{1}{2}$.

Definition 1: A transmission configuration is a vector $\bar{\tau}=$ $\left(\tau_{1}, \ldots, \tau_{N}\right)$ of $N$ integers that describes the packets transmitted by the sender for a given data block. Element $\tau_{j}$ in this vector indicates the number of packets transmitted using MCS- $j$.

The optimal 1-round transmission configuration is to transmit 3 MCS-2 packets, in which case the probability of the designated receiver to correctly decode the data block is $\left(\begin{array}{l}3 \\ 2\end{array}\right) \cdot \frac{1}{2}^{3}+\left(\begin{array}{l}3 \\ 3\end{array}\right) \cdot \frac{1}{2}^{3}=\frac{1}{2}$. The optimal 2-round protocol starts with a single MCS-2 packet. If the packet is correctly received by the designated receiver, the base station transmits a single MCS-1 packet in the next round. If the first transmission fails, the base station transmits two MCS-2 packets in the next round. The probability that the designated receiver will correctly decode the data block is $\frac{1}{2} \cdot(1-\epsilon)+\frac{1}{2} \cdot \frac{1}{2} \cdot \frac{1}{2}=\frac{5}{8}-\frac{\epsilon}{2}$, which is higher than for the 1-round optimal transmission configuration $\left(\frac{1}{2}\right)$.

For the rest of this subsection, we generalize the above example and show that when one uses MCS-1 and MCS-2 as defined above, the probability that the designated receiver will correctly decode the data block converges to 1 when the number of rounds increases. This is not a straightforward example when the bandwidth allocated for the transmission of each data block is limited.

Let $K=n+1$, and suppose that the available bandwidth $B$ is sufficient for transmitting $2 n+1$ MCS-2 packets or $n+0.5$ MCS-1 packets. The optimal 1-round transmission configuration is $2 n+1$ MCS-2 packets, in which case the probability that the designated receiver will correctly decode the data block is $\frac{1}{2}$.

The optimal $2 n+1$-round schedule is to transmit a single MCS-2 packet in every round until the number of packets correctly received by the designated receiver is strictly larger than the number of incorrectly received packets. Then, in the next (and last) round, the sender should transmit as many MCS-1 packets as possible. Let $r$ be the last transmission round of an MCS-2 packet. Since $r$ must be odd, let $r=2 k+1$. At the end of round $r$, there are $k+1$ correctly received packets, and $K-k-1=n+1-k-1=n-k$ more packets are required to correctly decode the data block. The remaining bandwidth is sufficient for transmitting $n-k$ MCS1 packets, which guarantees (with probability $1-\epsilon$ ) that the receiver will be able to correctly decode the data block. 
Denote the transmission results as a binary vector, where the $i$ th bit indicates whether the designated receiver correctly received the packet transmitted in the $i$ th round. The probability that the designated receiver will not be able to decode the data block is equal, up to an $\epsilon$, to the probability that every prefix of this vector will not contain more 1 s than 0 s. Note that in this case the vector is of size $2 n+1$ and all the packets are transmitted using MCS-2.

We now show that the number of binary vectors of size $2 n+1$ for which no prefix contains more 1 s than 0 s is $\left(\begin{array}{c}2 n+1 \\ n+1\end{array}\right)$. Let $A$ be the set of binary vectors of size $2 n+1$ that have $n+10$ s and $n 1$ s. Let $B$ be the set of binary vectors of size $2 n+1$ for which every prefix contains no more $1 \mathrm{~s}$ than $0 \mathrm{~s}$. Clearly, $|A|=\left(\begin{array}{c}2 n+1 \\ n+1\end{array}\right)$. We now present a bijection $f: A \rightarrow B$. Given $a \in A$, if $a \in B$ then $f(a)=a$. Otherwise, there is a prefix in $a$ that has more 1 s than 0s. Consider the following transformation $g$ on $a$ : find the shortest prefix that has more 0 s than 1 s and flip every bit in this prefix. Clearly, the number of $1 \mathrm{~s}$ decreases by exactly 1 . Note that $g$ is reversible (simply find the first prefix that holds more 0 s than 1 s and flip its bits). If $g(a) \in B$ then $f(a)=g(a)$; otherwise continue to apply $g$ on $a$ until receiving a vector that belongs to $B$. The function $f$ is bijective since $g$ is reversible and one can tell how many times $g$ has been applied by the total number of $1 \mathrm{~s}$. Thus, the number of vectors in $B$ is equal to the number of vectors in A.

Now, note that the probability to receive each of the $\left(\begin{array}{c}2 n+1 \\ n+1\end{array}\right)$ vectors is $\frac{1}{2^{2 n+1}}$. Thus, the probability that the designated receiver will correctly decode the data block is $1-\left[\left(\begin{array}{c}2 n+1 \\ n+1\end{array}\right) / 2^{2 n+1}\right]$. Since

$$
\begin{aligned}
& \left(\begin{array}{c}
2 n+1 \\
n+1
\end{array}\right)=\frac{(2 n+1) !}{(n+1) ! n !} \\
& \leq \frac{\sqrt{2}\left(\frac{2 n+1}{e}\right)^{2 n+1} \sqrt{2 \pi(2 n+1)}}{\left(\frac{n+1}{e}\right)^{n+1} \sqrt{2 \pi(n+1)}\left(\frac{n}{e}\right)^{n} \sqrt{2 \pi(n)}} \\
& \leq \frac{\left(\frac{2 n+1}{e}\right)^{2 n+1} \sqrt{(2 n+1)}}{\left(\frac{n+1}{e}\right)^{n+1} \sqrt{(n+1)}\left(\frac{n}{e}\right)^{n} \sqrt{\pi} \sqrt{n}} \\
& =\frac{2^{2 n+1}\left(n+\frac{1}{2}\right)^{n+1}\left(n+\frac{1}{2}\right)^{n} \sqrt{2} \sqrt{n+\frac{1}{2}}}{(n+1)^{n+1} \sqrt{(n+1)} n^{n} \sqrt{\pi} \sqrt{n}} \\
& \quad \leq\left(1+\frac{1}{2 n}\right)^{n} \frac{2^{2 n+1}}{\sqrt{n}} \leq \frac{2^{2 n+1} \sqrt{e}}{\sqrt{n}}
\end{aligned}
$$

we get that $1-\frac{\left(\begin{array}{c}2 n+1 \\ n+1\end{array}\right)}{2^{2 n+1}} \geq 1-O\left(\frac{1}{\sqrt{n}}\right)$, which converges to 1 as $n$ grows.

\section{E. 1-round RM-AMC(OC-1) is NP-hard}

We start by formally defining the 1-round RM-AMC(OC-1) problems:

\section{Problem 1 (1-round RM-AMC(OC-1)):}

Instance: The number $K$ of packets required to correctly decode a data block, an SNR for the designated receiver (the worst receiver in the designated group), an upper bound $B_{\max }$ on the bandwidth the sender can use for every data block, and a collection of $N$ MCSs: MCS-1, $\ldots, \operatorname{MCS}-N$. Each MCS- $j$ is a pair $\left(b_{j}, f_{j}\right)$, where $b_{j}$ is the bandwidth cost for transmitting a packet using MCS- $j$ and $f_{j}$ is the function that translates from an SNR value to the probability that a receiver with such an SNR will receive an MCS- $j$ packet with no error. Without loss of generality, we assume that $b_{j} \leq b_{k}$ holds for every $j<k$ and that $b_{1}=1$.

Objective: Find a transmission configuration such that the total bandwidth used for all the packets is not larger than $B_{\max }$ and the probability that the designated receiver will correctly decode the data block is maximized.

Theorem 1: The decision version of $\mathrm{RM}-\mathrm{AMC}(\mathrm{OC}-1)$ for 1-round is NP-hard.

The proof is presented in the Appendix.

\section{Algorithms FOR 1-ROUnd RM-AMC(OC-1)}

\section{A. Verifying the correctness of a solution}

We now show how the sender can efficiently check whether OC-1 holds for a given transmission configuration to the 1round $\mathrm{RM}-\mathrm{AMC}(\mathrm{OC}-1)$ problem. Let $t$ be the number of packets in the transmission configuration and $K$ be the number of packets a receiver needs to correctly decode a data block. Let $\operatorname{MCS}(h)$ be the index of the MCS used for the $h$ th packet in the transmission configuration. Let $V(h)$ be a vector with two elements: $V(h)=\left(p^{\operatorname{MCS}(h)}, 1-p^{\operatorname{MCS}(h)}\right)$, where $p^{\operatorname{MCS}(h)}$ is the probability that the designated receiver will correctly receive an $\operatorname{MCS}(h)$ packet. Denote by $\tilde{U}=\left(\tilde{u_{0}}, \ldots, \tilde{u}_{t}\right)$ the convolution of $V(1), \ldots, V(t) . \tilde{U}$ is a vector of length $t+1$, where $\tilde{u}_{l}, 0 \leq l \leq t$ is the probability that the designated receiver will correctly receive exactly $l$ packets. Hence, the probability that this receiver will correctly decode the data block is $\sum_{l=K}^{t} \tilde{u}_{l}$.

To efficiently compute the convolution of $V(1), \ldots, V(t)$, we divide this set of vectors into 2 equal sets. We recursively compute the convolution of the vectors in each of the 2 sets and get two new vectors. Then, we compute the convolution of the returned new vectors. We use the fact that the convolution of 2 vectors with size $n$ can be computed in $O(n \cdot \log (n))$ using Fast Fourier Transform [6]. Hence, each recursive step takes $O(t \cdot \log (t))$ time and the total computation takes $O\left(t \cdot \log ^{2}(t)\right)$.

The $O\left(t \cdot \log ^{2}(t)\right)$ computational complexity can be improved using the following observation. When the convolution of two vectors creates a vector with more than $K$ elements, the resulting vector can be replaced by a short vector with exactly $K$ elements. The first $K-1$ elements of the short vector are identical to those of the long one. The $K$ th element is set to $\sum_{i \geq K} y_{i}$, where $y_{i}$ is the $i$ th element of the long vector. Consequently, the $K$ th element indicates the probability that the designated receiver will be able to correctly decode the data block. The information we lose in this process, namely, how many packets the designated receiver will be able to decode in addition to the required $K$ packets, is not relevant.

If $T(x)$ is the time required for computing the convolution 
of $x$ short vectors, then the following recursive equation holds:

$$
T(x) \leq \begin{cases}2 \cdot T(x / 2)+x \log x & \text { if } x<K \\ 2 \cdot T(x / 2)+K \log K & \text { Otherwise. }\end{cases}
$$

Thus, for $t \geq K$ we get $T(t)=O\left(t \cdot \log (K)+K \cdot \log ^{2}(K)\right)$.

\section{B. An optimal algorithm for 1-round RM-AMC $(O C-1)$ with a small number of MCSs}

Definition 2: An MCS is said to be unacceptable for a given $S N R$ if the probability that a packet will be correctly received by a receiver with such an SNR is almost 0 .

Definition 3: MCS-1 is said to dominate MCS-2 for a given SNR if the probabilities that a receiver with such an SNR will correctly receive an MCS-1 packet and an MCS-2 packet are almost identical, but the bandwidth used for transmitting an MCS-1 packet is smaller than that used for transmitting an MCS-2 packet.

A transmission configuration that uses an unacceptable MCS is not optimal because the contribution of the packets transmitted using this MCS does not justify their bandwidth cost. A transmission configuration that uses a dominated MCS is not optimal because it can be replaced with the dominating MCS that uses less bandwidth without affecting the probability that a receiver will correctly decode the data block.

In many practical applications, there are at most 3 MCSs that are acceptable and are not dominated by other MCSs. For such applications a brute-force search is sufficient. Therefore, Algorithm 1 can be used to find an optimal solution for 1round $\mathrm{RM}-\mathrm{AMC}(\mathrm{OC}-1)$.

Algorithm 1: (an optimal algorithm for 1-round RM$\mathrm{AMC}(\mathrm{OC}-1)$ with a small number of MCSs)

1) Set the list $L_{p}$ to contain all possible transmission configurations whose bandwidth $\leq B_{\max }$.

2) Find in $L_{p}$ the transmission configuration $\bar{m}$ that maximizes the probability that the designated receiver will correctly decode the data block, and store it in solval.

3) Return solval.

The running time of Algorithm 1 is $O\left(\beta \cdot\left(B_{\max }\right)^{N}\right)$ where $\beta$ is the time complexity for verifying that OC-1 holds and $N$ is the number of MCSs.

\section{A heuristic for 1-round RM-AMC(OC-1) based on the Unbounded Knapsack Problem}

We now present a heuristic for 1-round RM-AMC(OC-1), based on a reduction to the Unbounded Knapsack Problem (UKP) [12]. UKP is an extension of USSP [12]. The instance is a set $S$ of item types $s_{1}, s_{2}, \ldots, s_{m}$ and a capacity $C$. Each type $s_{i}$ has a weight $w\left(s_{i}\right)$ and a profit $p\left(s_{i}\right)$. The objective is to find a vector $S^{\prime}=\left(s_{1}^{\prime}, \ldots, s_{m}^{\prime}\right)$ of items whose aggregated profit $\sum_{i=1}^{m} s_{i}^{\prime} \cdot p\left(s_{i}\right)$ is maximum and whose aggregated weight $\sum_{i=1}^{m} s_{i}^{\prime} \cdot w\left(s_{i}\right)$ is not larger than $C$.

To reduce an instance of this problem to an instance of UKP, each MCS is represented by an item type, and the bandwidth limitation $B_{\max }$ is translated into the capacity $C$. The weight of a type is the bandwidth cost of the corresponding MCS, and the profit of each type is the probability that a packet of the corresponding MCS will be correctly received by the designated receiver. To transform a solution $S^{\prime}=\left(s_{1}^{\prime}, \ldots, s_{m}^{\prime}\right)$ for the reduced UKP problem to a solution for RM-AMC (OC1 ), we construct a transmission configuration with $s_{i}^{\prime}$ packets transmitted using MCS- $i$ for every $i$.

Observation 1: The expected number of correctly received packets for a given transmission configuration in the 1-round $\mathrm{RM}-\mathrm{AMC}(\mathrm{OC}-1)$ problem is equal to the aggregated profit in the corresponding UKP problem.

UKP has a simple 2-approximation greedy algorithm whose running time is $O(m \cdot \log (m))$ using sorting and $O(m)$ using linear selection [12]. It also has a pseudopolynomial timeoptimal dynamic programming algorithm whose running time is $O(m \cdot C)$ [12] and an FPTAS [12].

When the number of MCSs is small, the number of UKP types is also small. Small instances can be optimally solved in polynomial time [15]. This gives rise to the following heuristic for the 1-round RM-AMC(OC-1) problem.

Algorithm 2: (A heuristic for 1-round RM-AMC(OC-1) with a large number of MCSs)

1) Reduce the 1-round $\mathrm{RM}-\mathrm{AMC}(\mathrm{OC}-1)$ instance to an UKP instance as described above.

2) Run an algorithm for finding a solution $S^{\prime}=$ $\left(s_{1}^{\prime}, \ldots, s_{m}^{\prime}\right)$ for the UKP instance.

3) Translate $S^{\prime}$ to a solution for 1-round RM-AMC(OC-1), where the number of packets transmitted using MCS- $i$ is $s_{i}^{\prime}$.

The running time of Algorithm 2 is equal to the running time of the algorithm used to solve the UKP problem in step 2. Note, however, that Algorithm 2 has no performance guarantee even if UKP is solved optimally. To see this, consider two MCSs: MCS-1 and MCS-2. Suppose that a packet encoded using MCS-1 requires twice the bandwidth required by MCS2. On the other hand, suppose that the probability that the designated receiver will correctly receive an MCS-1 packet is $1-\epsilon$, and the probability that it will correctly receive an MCS-2 packet is $\frac{1}{4}$. Suppose that the available bandwidth $B$ is sufficient for transmitting 1 MCS-1 packet or 2 MCS-2 packets and that $K=2$. In this case the transmission configuration returned by Algorithm 2 is composed of a single MCS1 packet. Consequently, the probability that the designated receiver will correctly decode the data block is 0 . In contrast, the optimal transmission configuration for this instance is to send 2 MCS-2 packets, which results in probability $\frac{1}{16}$.

The table in Figure 1 summarizes the algorithms proposed in this section.

\section{Extending RM-AMC(OC-1) To Multiple Rounds}

We now describe how to extend 1-round RM-AMC(OC-1) to multiple rounds.

\section{$A$. The R-rounds $R M-A M C(O C-1)$ problem}

The $R$-rounds $\mathrm{RM}-\mathrm{AMC}(\mathrm{OC}-1)$ problem is similar to the 1-round RM-AMC(OC-1), except that there are up to $R$ transmission rounds for the same data block. The number of rounds $R$ is chosen in advance to meet the delay constraint. 


\begin{tabular}{|c|c|c|c|}
\hline Problem & Algorithm & Performance & Time complexity \\
\hline \hline \multirow{2}{*}{ 1-round RM-AMC(OC-1) } & Alg. 1 & Optimal & $O\left(\beta \cdot\left(B_{\max }\right)^{N}\right)$ \\
\cline { 2 - 4 } & Alg. 2 & Heuristic & The time for solving the reduced UKP problem \\
\hline \multirow{2}{*}{$R$-round RM-AMC(OC-1) } & Alg. 3 & Optimal & $O\left(\left(B_{\max }\right)^{(N+3)} \cdot K \cdot R\right)$ \\
\cline { 2 - 4 } & Alg. 4 & Heuristic & $O\left(\left(B_{\max }\right)^{3} \cdot N \cdot K \cdot R\right)$ \\
\hline $\begin{array}{c}\text { RM-AMC(OC-1) } \\
\text { with an unbounded number of rounds }\end{array}$ & Alg. 5 & Optimal & $O\left(N \cdot K \cdot B_{\max }\right)$ \\
\hline
\end{tabular}

Fig. 1. The various algorithms proposed for RM-AMC(OC-1)

If the application can tolerate a higher delay, the sender will use a larger value of $R$. This will increase the probability for successfully decoding the data block for a given value of $B_{\max }$. After every round of transmission, the sender receives a feedback message about the number of packets correctly received by the designated receiver during this round. Since the base station does not know which node is the designated receiver, it should run an algorithm similar to that proposed by NORM [2], where a receiver reports the number of missing packets only if this report is not superseded by the reports already sent by other receivers.

We now formally define the $R$-round RM-AMC $(\mathrm{OC}-1)$ problem:

\section{Problem 2 ( $R$-round RM-AMC(OC-1)):}

Instance: The same as for 1-round RM-AMC (OC-1).

Objective: Find a transmission configuration to be used in each round, based on the outcome of previous rounds, such that the total bandwidth used is not larger than $B_{\max }$ and the probability that the designated receiver will correctly decode the data block is maximized.

Implementation Note: In practice, no node is "nominated" as the designated receiver. A practical way for the base station to know which node is the designated receiver is to ask those receivers whose SNR is above the desired threshold (i.e., the designated group) to report how many packets they are missing and their SNR. (This is done after the first round of packets is broadcast.) Each such receiver draws a random backoff time from a truncated exponential distribution. The random backoff time depends also on the SNR, such that a receiver with a larger SNR will be likely to wait longer. A receiver whose timer expires checks whether its SNR is smaller than the smallest SNR reported so far. (Thus, each feedback sent by a receiver on the uplink should be reflected by the base station on the downlink.) If it is not smaller, the receiver suppresses its feedback. If it is smaller, the receiver sends a feedback message that contains its SNR and the number of missing packets. The last reporting receiver is considered to be the designated receiver. If more than 2 rounds are necessary, this receiver will be explicitly queried by the base station in the next feedback rounds.

Theorem 2: The decision version of $R$-round RM$\mathrm{AMC}(\mathrm{OC}-1)$ is NP-hard ${ }^{1}$.

The proof is presented in the Appendix.

\footnotetext{
${ }^{1}$ Following Theorem 1, this theorem is trivial for an arbitrary $R$. However, here we consider a constant $R>1$ that is known in advance and is not a part of the input.
}

Observation 2: From the proof of Theorem 2 it follows that for every solution for the $R$-round RM-AMC(OC-1) problem with $K=1$, there is a solution with the same performance guarantee and the same bandwidth limitation that uses only a single round. The only benefit in using more than a single round in this case $(K=1)$ is the possible reduction in total bandwidth cost.

\section{B. An optimal algorithm for R-round RM-AMC(OC-1) with a} small number of MCSs

Let $G^{\bar{\tau}}[\geq k]$ be the probability that at least $k$ packets will be correctly received by the designated receiver for a transmission configuration $\bar{\tau}$ in 1-round RM-AMC(OC-1), and let $G^{\bar{\tau}}[k]$ be the probability that exactly $k$ packets will be correctly received. In Section IV-A we showed how to find $G^{\bar{\tau}}[\geq k]$ and $G^{\bar{\tau}}[k]$. We now assume their values are given in an $O\left(\left(B_{\max }\right)^{N} \cdot K\right)$ size array, where $B_{\max }$ is the total bandwidth allowed for the transmission of the data block, $N$ is the number of available MCSs, and $K$ is the number of packets required for decoding the data block. Let $H(k, b, r)$ be the maximum probability that the receiver will correctly receive at least $k$ packets using a protocol of $r$ rounds whose total bandwidth consumption is $b$, and let $T_{b}$ be the set of all transmission configurations whose bandwidth consumption is $b$. We now define the following equation for computing $H\left(K, B_{\max }, R\right)$ using dynamic programming:

$$
\begin{aligned}
& H(k, b, 1)=\max _{\bar{\tau} \in T_{b}} G^{\bar{\tau}}[\geq k], \\
& H(k, b, r) \text { is the maximum of } \\
& \sum_{i=0}^{c} G^{\bar{\tau}}[i] \cdot H(\max (k-i, 0), b-c, r-1)
\end{aligned}
$$

obtained for every transmission configuration $\bar{\tau} \in T_{c}$

where $0 \leq c \leq b$.

Theorem 3: Eq. 2 calculates $H(k, b, r)$ as defined earlier. The proof is presented in the Appendix.

There are $K \cdot B_{\max } \cdot R$ entries to compute. Each entry takes $O\left(\sum_{i=0}^{B_{\max }}\left|T_{i}\right| \cdot B_{\max }\right)=O\left(\left(B_{\max }\right)^{(N+2)}\right)$ time. Therefore, the total time complexity is $O\left(\left(B_{\max }\right)^{(N+3)} \cdot K \cdot R\right)$. To return the transmission configuration that corresponds to the value of $H(k, b, r)$, we create an array $T$. During the computation of $H(k, b, r)$ we update entry $T[k, b, r]$ to contain the transmission configuration used to obtain the value of $H(k, b, r)$. This idea is summarized in the following algorithm. 
Algorithm 3: (An optimal algorithm for $R$-round RM$\mathrm{AMC}(\mathrm{OC}-1)$ with a small number of MCSs)

1) Using Eq. 2 and dynamic programming, compute $H(k, b, r)$ for $0 \leq k \leq K, 0 \leq b \leq B_{\max }, 1 \leq r \leq R$.

2) curk $\leftarrow K$, curb $\leftarrow B_{\max }$.

3) For $i=1$ to $R$ :

a) Use $T[$ curk, curb, $R-i+1]$ as the transmission configuration in the $i$ th round and subtract its bandwidth cost from curb.

b) After getting a feedback message about the outcome of the previous round, subtract from curk the number of packets correctly received by the designated receiver in the $i$ th round.

\section{A heuristic for R-round RM-AMC (OC-1) with a large number of MCSs}

When the value of $N$ is larger than 2-3 or the value of $B_{\max }$ is in the order of several hundreds, the running time complexity of Algorithm 3 renders it impractical. We now describe a heuristic whose running time is much better.

In the beginning of every round, the algorithm is given the remaining bandwidth and the number of packets the designated receiver has already correctly received. The algorithm returns the transmission configuration for this round.

During every step of its execution the algorithm determines: (a) the amount of bandwidth to be used in the next round, and (b) whether to use this bandwidth as an input to Algorithm 2 or to use it for a transmission configuration that contains a single MCS. If Algorithm 2 is used in every round and the UKP problem in Algorithm 2 is solved optimally, the solution produced by the heuristic has the same probability as a solution for a single round with the same $B_{\max }$. We will see, in Section VI, that combining Algorithm 2 with an algorithm that uses a single MCS is a good heuristic for 1-round, and therefore it makes sense to use a similar rationale for multiple rounds.

Let $\overline{\tau_{b}}$ be the transmission configuration returned by Algorithm 2 when running with $B_{\max }=b$. Let $\overline{\tau_{b}^{j}}$ be the transmission configuration containing only MCS $-j$ packets that uses the maximum possible bandwidth under bandwidth limitation $b$. Recall that $G^{\bar{\tau}}[\geq k]$ is defined as the probability that at least $k$ packets will be correctly received by the designated receiver for a transmission configuration $\bar{\tau}$ in 1-round RM-AMC(OC1 ), and $G^{\bar{\tau}}[k]$ is defined as the probability that exactly $k$ packets will be correctly received.

We also define $U_{b}[k]$ and $S_{b}^{j}[k]$ as follows:

$$
\begin{aligned}
& U_{b}[k]= \begin{cases}G^{\overline{\tau_{b}}}[k] & \text { if } \overline{\tau_{b}} \text { uses bandwidth } b \\
0 & \text { Otherwise }\end{cases} \\
& S_{b}^{j}[k]= \begin{cases}G^{\overline{\tau_{b}^{j}}}[k] & \text { if } \overline{\tau_{b}^{j}} \text { uses bandwidth } b \\
0 & \text { Otherwise. }\end{cases}
\end{aligned}
$$

Similarly, we define $U_{b}[\geq k]$ and $S_{b}^{j}[\geq k]$ as follows:

$$
U_{b}[\geq k]= \begin{cases}G^{\overline{\tau_{b}}}[\geq k] & \text { if } \overline{\tau_{b}} \text { uses bandwidth } b \\ 0 & \text { Otherwise }\end{cases}
$$

$$
S_{b}^{j}[\geq k]= \begin{cases}G^{\overline{\tau_{b}^{j}}}[\geq k] & \text { if } \overline{\tau_{b}^{j}} \text { uses bandwidth } b \\ 0 & \text { Otherwise. }\end{cases}
$$

When $U_{b}[k]$ and $U_{b}[\geq k]$ are computed using Eq. 3 and Eq. 4, $\overline{\tau_{b}}$ is found using Algorithm 2.

Let $M(k, b, r)$ be the maximum probability that the designated receiver will correctly receive at least $k$ packets using an $r$-round algorithm whose total bandwidth consumption is $b$ when we use in every round Algorithm 2 or a single MCS. We now define the following equations for computing $M\left(K, B_{\max }, R\right)$ using dynamic programming:

$$
M(k, b, 1)=\max \left\{U_{b}[\geq k], S_{b}^{1}[\geq k], \ldots, S_{b}^{N}[\geq k]\right\}
$$

$$
M(k, b, r)=\max \left\{\begin{array}{ll}
\sum_{i=0}^{c} U_{c}[i] \cdot & \sum_{i=0}^{c} S_{c}^{j}[i] \cdot \\
M(\max (k-i, & M(\max (k-i, \\
0), b-c, r-1) & 0), b-c, r-1)
\end{array}\right\}
$$

for $1 \leq j \leq N$ and $0 \leq c \leq b$.

Theorem 4: Eq. 5 calculates $M(k, b, r)$ as defined earlier. The proof is presented in the Appendix.

Note that in the computation of $M(k, b, r)$ there are $N+1$ elements from which the maximum is taken. There are $K \cdot B_{\max } \cdot R$ entries to compute. Each entry takes $O\left(\left(B_{\max }\right)^{2} \cdot N\right)$ time. Assuming that Algorithm 2 is solved using a 2-approximation polynomial time algorithm [12], the total time complexity is $O\left(\left(B_{\max }\right)^{3} \cdot N \cdot K \cdot R\right)$. Using a similar idea to that presented in Section $\mathrm{V}$-B, we create an array $T$ whose $[k, b, r]$ entry contains the transmission configuration used to obtain the value of $M(k, b, r)$. We now summarize the whole algorithm.

Algorithm 4: (A heuristic for $R$-round RM-AMC(OC-1) with a large number of MCSs)

1) Use dynamic programming to compute $M(k, b, r)$ for $0 \leq k \leq K, 0 \leq b \leq B_{\max }, 1 \leq r \leq R$, according to Eq. 5 .

2) curk $\leftarrow K$, curb $\leftarrow B_{\max }$.

3) For $\mathrm{i}=1$ to $R$ :

a) Use $T[$ curk, curb, $R-i+1]$ as the transmission configuration in the $i$ th round and subtract the bandwidth cost of this transmission configuration from curb.

b) After getting a feedback message about the outcome of the previous round, subtract from curk the number of packets correctly received by the designated receiver in the $i$ th round.

\section{Unbounded number of rounds}

The case where the number of rounds is unbounded is interesting not only because of the theoretical analysis, but also because it allows us to find the number of rounds for which the performance is very close to the maximum possible with an unbounded number of rounds. Since the bandwidth limit 
still holds, the number of rounds is, in practice, limited by the maximum number of packets the sender can send. Thus, the unbounded number of rounds is equivalent to $R$-rounds RM-AMC(OC-1) with $R=B_{\max }$.

Observation 3: Every optimal transmission configuration for the RM-AMC(OC-1) with an unbounded number of rounds that uses more than one packet in any round can be replaced by an optimal transmission configuration that uses exactly one packet in every round.

Let $F(k, b)$ be the maximum probability that at least $k$ packets will be correctly received by the designated receiver using a bandwidth cost of $b$. The following equation is used for computing $F\left(K, B_{\max }\right)$ using dynamic programming.

$$
\begin{gathered}
F(k, 0)= \begin{cases}0 & \text { if } k>0 \\
1 & \text { Otherwise. }\end{cases} \\
F(k, b)= \begin{cases}F(k, 0) & \text { if } \forall j, b_{j}>b \\
\max _{b_{j} \leq b}\left(\left(p^{j} \cdot F\left(k-1, b-b_{j}\right)+\right.\right. & \\
\left.\left.\left(1-p^{j}\right) \cdot F\left(k, b-b_{j}\right)\right)\right) & \text { Otherwise. }\end{cases}
\end{gathered}
$$

Theorem 5: Eq. 6 calculates $F(k, b)$ as defined earlier. The proof is presented in the Appendix.

The computation of each entry requires $O(N)$ operations and the total number of entries is $O\left(K \cdot B_{\max }\right)$. Thus, the total running time is $O\left(N \cdot K \cdot B_{\max }\right)$.

During the computation of $F(k, b)$ we update entry $A[k, b]$ to contain the MCS used to obtain the value of $F(k, b)$. From Observation 3 we note that there exists an optimal solution for the RM-AMC(OC-1) problem with an unbounded number of rounds that uses a single packet whose MCS is $A[i, b]$ in every round. The value of $i$ indicates the number of packets the designated receiver has to receive in order to correctly decode the data block. It is equal to $K$ minus the number of packets correctly received in all previous rounds. The value of $b$ indicates the bandwidth available for transmission of this data block, namely, $B_{\max }$ minus the bandwidth used in previous rounds.

We now present an optimal algorithm for RM-AMC(OC-1) with an unbounded number of rounds.

Algorithm 5: (An optimal algorithm for RM-AMC(OC-1) with an unbounded number of rounds)

1) Use dynamic programming to compute $F(k, b)$ for $0 \leq$ $k \leq K, 0 \leq b \leq B_{\max }$, according to Eq. 6.

2) curk $\leftarrow K$, curb $\leftarrow B_{\max }$.

3) While curk $>0$ and curb $\geq b_{1}$ (i.e., the designated receiver has not yet correctly received $K$ packets and there is enough bandwidth for a new packet) do:

a) In the $i$ th round, transmit 1 packet using the MCS indicated in $A[c u r k, c u r b]$ and subtract the bandwidth cost of this packet from curb.

b) If the designated receiver correctly receives the last transmitted packet, curk $\leftarrow$ curk -1 .

Since there are at most $B_{\max }$ rounds, the running time of Algorithm 5 is $O\left(N \cdot K \cdot B_{\max }+B_{\max }\right)=O\left(N \cdot K \cdot B_{\max }\right)$.

\section{Simulation Study of the Various Algorithms}

The goal of this section is threefold:

- To compare the benefit of using multiple MCSs for the considered reliable multicast application to the current practice of using only one MCS, for OC-1.

- To compare the performance of the various algorithms presented in this paper for OC- 1 .

- To evaluate the benefit of using multiple rounds.

Throughout this section we consider 7 possible MCSs. These MCSs and the corresponding probabilities of the designated receiver to correctly receive a packet for a certain SNR are computed according to [4]. To compare the results of using multiple MCSs with those of a single MCS, we now present the optimal single MCS algorithm:

Algorithm 6: (an optimal algorithm for 1-round RM$\mathrm{AMC}(\mathrm{OC}-1)$ with a single $\mathrm{MCS})$

1) For every MCS, build a transmission configuration with as many packets as can be accommodated using a bandwidth of $B_{\max }$.

2) From all these transmission configurations, use the one that maximizes the probability that the designated receiver will correctly decode the data block.

The time complexity of Algorithm 6 is $O\left(N \cdot B_{\max } \cdot \beta\right)$.

The results reported in this section are for SNR values between $6 \mathrm{~dB}$ and $10 \mathrm{~dB}$. However, we saw similar results for different SNR values. Some of our graphs show the probability that the designated receiver will correctly decode the data block vs. the SNR it experiences. The SNR values displayed in those graphs are between $6 \mathrm{~dB}$ and a value for which the success probability is very close to 1 ( $>0.999)$, because increasing the SNR value further does not affect the success probability. In the considered SNR range, there are up to 4 relevant MCSs, for which the success probability is greater than 0 .

Throughout this section we consider $K=6$ packets per data block. We saw no substantial differences when we increased $K$ to 10 . For every SNR value, we set $B_{\max }$ to be sufficient for 5 packets of the MCS that consumes the highest bandwidth, plus 1 packet of the MCS that consumes the second-highest bandwidth. Figure 2 shows the probability that the designated receiver will correctly decode the data block vs. the SNR it experiences for three algorithms: Algorithm 1 (optimal), Algorithm 2 and Algorithm 6 (optimal for 1 MCS). For Algorithm 2 we used an optimal pseudopolynomial algorithm to solve the UKP problem. However, solving UKP using the greedy algorithm instead of the optimal pseudopolynomial algorithm only slightly reduces the performance of Algorithm 2.

Algorithm 2 performs very much like Algorithm 1 (the optimal algorithm) and both are represented by a single curve. When we use a single MCS, the performance is significantly worse. This is because the value of $B_{\max }$ is not large enough for transmitting 6 packets using the best MCS for the designated receiver.

In Figure 3 and Figure 4 we concentrate on a single SNR, of $7.5 \mathrm{~dB}$, but consider different $B_{\max }$ values. In both graphs we 


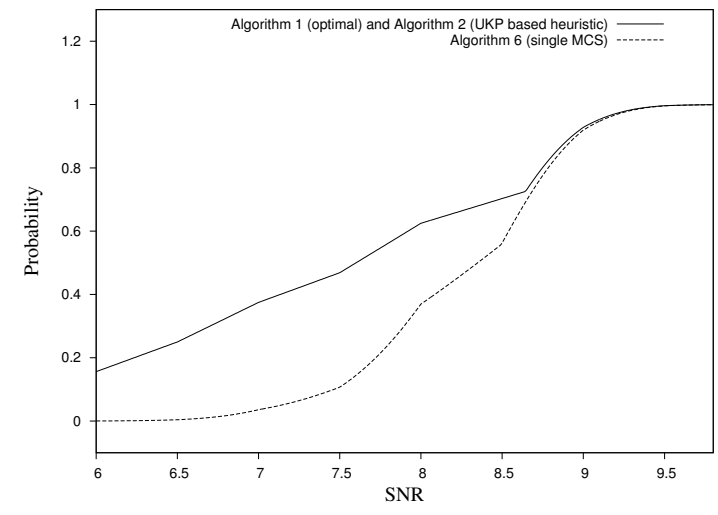

Fig. 2. Probability that the designated receiver will correctly decode a data block vs. the SNR it experiences for Algorithm 1, 2 and 6

show the probability that the designated receiver will correctly decode the data block vs. $B_{\max }$. In Figure 3 we see that Algorithm 2 performs very much like Algorithm 1 for most of the $B_{\max }$ values. However, there is a range of $B_{\max }$ where Algorithm 2 is suboptimal because it sends fewer than 6 packets, which results in a probability 0 that the designated receiver will correctly decode the data block.

In Figure 4 we see that Algorithm 6 (the optimal single MCS algorithm) performs very much like Algorithm 1 exactly in the same $B_{\max }$ values for which Algorithm 2 performs poorly. This is because Algorithm 6 uses at least 6 packets as soon as the bandwidth allows it.

We conclude that for 1-round RM-AMC(OC-1) with a small number of MCSs, Algorithm 1 is recommended. For more than 3 MCSs, running both Algorithms 2 and 6 is recommended. From the two returned transmission configurations, the one that maximizes the probability for the designated receiver to correctly decode the data block should be chosen. This algorithm will be close to optimal for all $B_{\max }$ values, and its time complexity is equal to the time complexity of Algorithm 2. In addition, in Algorithm 2 the greedy 2-approximation procedure is sufficient for solving the UKP problem.

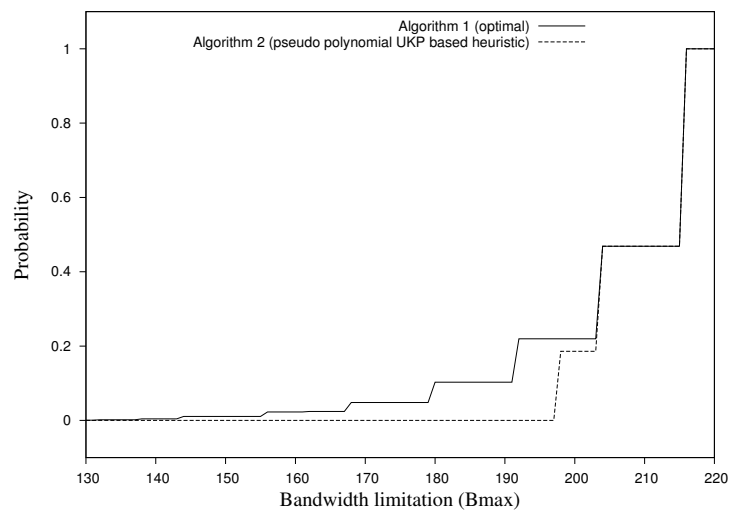

Fig. 3. Probability that the designated receiver will correctly decode a data block vs. the bandwidth limitation for Algorithm 1 and 2

We now present simulation results for multiple rounds. We used $K=6$, and set $B_{\max }$ to be sufficient for exactly 5

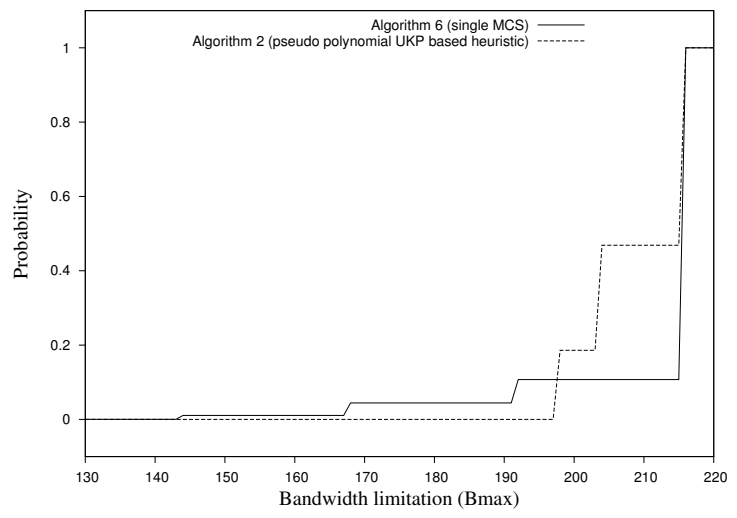

Fig. 4. Probability that the designated receiver will correctly decode a data block vs. the bandwidth limitation for Algorithm 2 and 6

packets of the most bandwidth consuming MCS (for every considered SNR) and 1 packet of the second most bandwidth consuming MCS. We compare the performance of 1 round to 2 rounds and to an unbounded number of rounds. For 1 round we used Algorithm 1, for 2 rounds we used Algorithm 3 with $R=2$, and for the theoretical unbounded number of rounds we used Algorithm 5. Recall that all these algorithms are optimal.

Figure 5 shows the probability that the designated receiver will correctly decode the data block vs. the SNR it experiences. We see that the 2-round protocol performs better than the 1round protocol and very close to the unbounded number of rounds protocol.

We increased $K$ to 30 , and set $B_{\max }$ to be sufficient for 29 packets of the most bandwidth consuming MCS and 1 packet of the second most bandwidth consuming MCS. The results are depicted in Figure 6. We see that for larger values of $K$, the benefit of using more rounds increases. This is because the bandwidth used by the sender for each data block is greater, which allows more combinations of MCSs using the information collected during every round.

Another interesting case is when $K=30$ and $B_{\max }$ is sufficient for $5 \cdot 5=25$ packets of the most bandwidth consuming MCS and $1 \cdot 5=5$ packets of the second most bandwidth consuming MCS. The results for this case are depicted in Figure 7. Compared to Figure 6, the success probability is smaller for SNRs $<8.5 \mathrm{~dB}$. The reason is that less bandwidth is used and therefore more packets are transmitted using the second most bandwidth consuming MCS. For low SNRs, the probability to receive such a packet is very small. As the SNR increases, the probability increases until it reaches 1 for SNR of $9 \mathrm{~dB}$, because for this SNR value the probability to receive a packet transmitted using the second most bandwidth consuming MCS is close to 1 . We can see that for this case the benefit of using more rounds is greater than for the case where $K=6$.

We also compared the performance of 1 round, 2 rounds, and the theoretical unbounded number of rounds with different values of $B_{\max }$ for $K=6$ and constant SNR of $8.5 \mathrm{~dB}$. The results, depicted in Figure 8, are similar to those reported in Figure 5. 


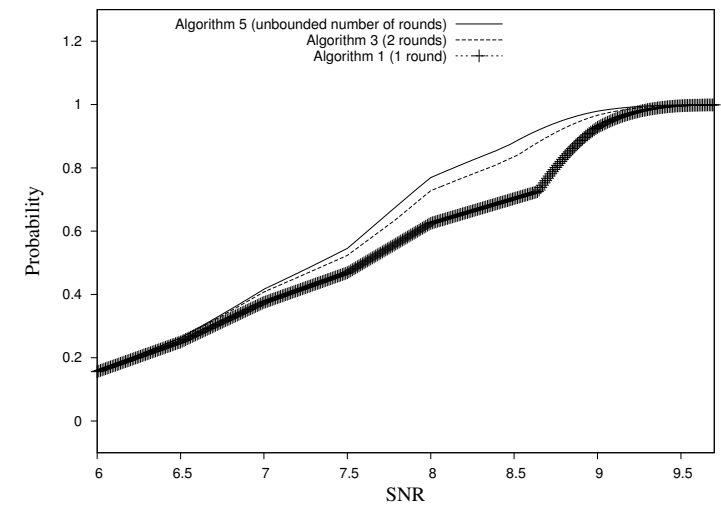

Fig. 5. Probability that the designated receiver will correctly decode a data block vs. the SNR it experiences for Algorithm 1, 3 and 5 with $K=6$

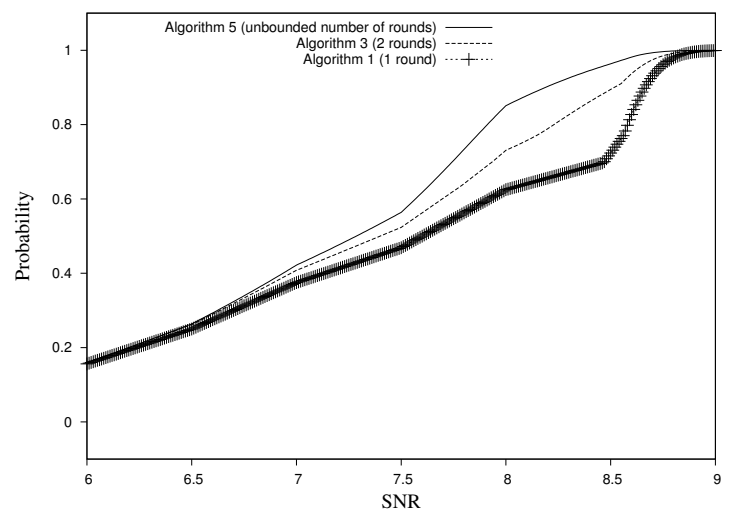

Fig. 6. Probability that the designated receiver will correctly decode a data block vs. the SNR it experiences for Algorithm 1, 3 and 5 with $K=30$ when $B_{\max }$ is sufficient for 29 packets of the most bandwidth consuming MCS and 1 packet of the second most bandwidth consuming MCS

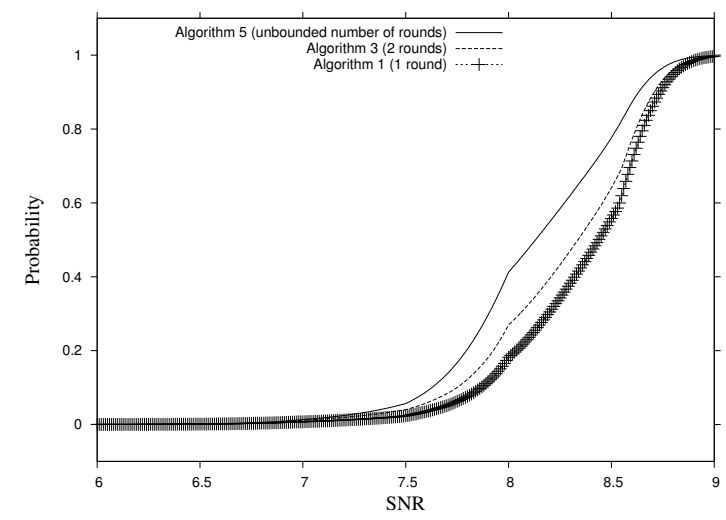

Fig. 7. Probability that the designated receiver will correctly decode a data block vs. the SNR it experiences for Algorithm 1, 3 and 5 with $K=30$ when $B_{\max }$ is sufficient for 25 packets of the most bandwidth consuming MCS and 5 packets of the second most bandwidth consuming MCS

We also compared the performance of Algorithm 3 to the performance of Algorithm 4 with 2 rounds, for $K=6$ and $K=30 . B_{\max }$ is set to be sufficient for $K-1$ packets of the most bandwidth consuming MCS and 1 packet of the second most bandwidth consuming MCS. Despite the fact that

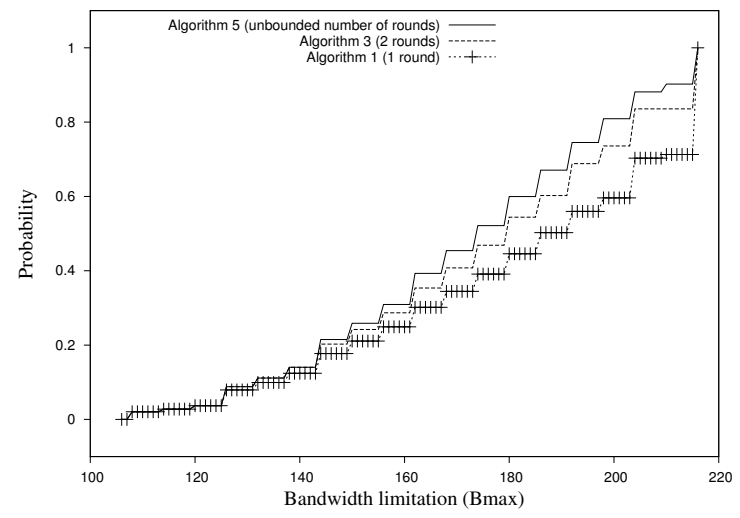

Fig. 8. Probability that the designated receiver will correctly decode a data block vs. the bandwidth limitation for Algorithm 1, 3 and 5

Algorithm 3 is optimal while Algorithm 4 is only heuristic, we found no difference in their performance. We made a similar observation when we used other sets of parameters.

In summary, we saw that using multiple MCSs improves the performance for OC-1. We also saw that when the optimal algorithm (Algorithm 1) cannot be used, Algorithm 2 is the second best. In addition, we saw that increasing the number of rounds from 1 to 2 for OC-1 improves the performance significantly for some SNR values. However, increasing the number of rounds further adds no significant improvement. Finally, we saw that the polynomial time heuristic for multiple rounds (Algorithm 4) yields results similar to those of the pseudopolynomial optimal algorithm.

\section{EXTENSIONS TO OTHER OPTIMIZATION CRITERIA}

While OC-1 is an important optimization criterion for reliable multicast, other optimization criteria are relevant as well. In this section we describe two such criteria:

OC-2 Minimize the total bandwidth, while guaranteeing that the probability of every receiver from the designated group to decode a data block is $\geq P$.

OC-3 Like OC-2, except that $P$ might be different for different subgroups. For example, one may define two subgroups: one with a good channel and one with a bad one, and assign to the first subgroup a higher target probability.

We note that all the problems defined earlier, except for the unbounded number of rounds case, are NP-hard for OC-2 and OC-3 as well. To see why, observe that the decision version of $\mathrm{RM}-\mathrm{AMC}(\mathrm{OC}-2)$ is identical to the decision version of RM$\mathrm{AMC}(\mathrm{OC}-1)$, and therefore RM-AMC(OC-2) is also NP-hard. To prove that OC-3 is also NP-hard, it is sufficient to show that every OC-2 instance can be reduced to an OC-3 instance. The reduction is trivial: select for every receiver the same probability threshold considered for OC-2.

That OC-2 holds for a given transmission configuration can be verified using our OC-1 verification procedure, described in Section IV-A. To verify that OC-3 holds for a given transmission configuration, we divide the receivers into groups according to the OC-3 thresholds. Then, we verify that OC-2 holds for each subgroup. 
In practical applications there exists an MCS for which the probability of the receiver with the worst SNR in the designated group to successfully receive a packet encoded using this MCS is very close to 1 . Let $b_{m}$ be the bandwidth cost of a packet sent using this MCS. Transmitting only $K$ packets using this MCS guarantees, with probability very close to 1 , that every receiver in the designated group of OC-2 and OC-3 will successfully receive the data block. Therefore, an optimal solution for RM-AMC(OC-2) and RM-AMC(OC-3) will have a bandwidth $\leq K \cdot b_{m}$. This rationale can be used for finding an optimal solution for OC-2/OC-3 using the following algorithm:

1) Let $L_{p}$ contain all possible transmission configurations whose bandwidth $\leq K \cdot b_{m}$.

2) Find in $L_{p}$ the transmission configuration $\bar{m}$ that satisfies OC-2/OC-3 and has the minimal bandwidth requirements. Store it in solval.

3) Return solval.

The running time of this algorithm is $O\left(\beta \cdot\left(K \cdot b_{m}\right)^{N}\right)$.

As we did for the optimal single MCS algorithm for OC-1 (Algorithm 6), we can also define polynomial time optimal single MCS algorithms for OC-2 and OC-3, as follows:

1) For every MCS, build a transmission configuration with as many packets as can be accommodated using a bandwidth of $\leq K \cdot b_{m}$.

2) From all these transmission configurations, choose the one that minimizes the bandwidth and satisfies the relevant probability threshold(s).

We compared the performance of the OC-2 optimal algorithm and the OC-2 single MCS algorithm for $K=6$ and $K=10$ with probability threshold of $P=0.99$. The results are depicted in Figure 9, which describes the bandwidth used for transmitting a data block vs. the SNR. It turns out that both algorithms have the same performance for this set of parameters, as well as for every other set we used. Therefore, the graph shows only one curve.

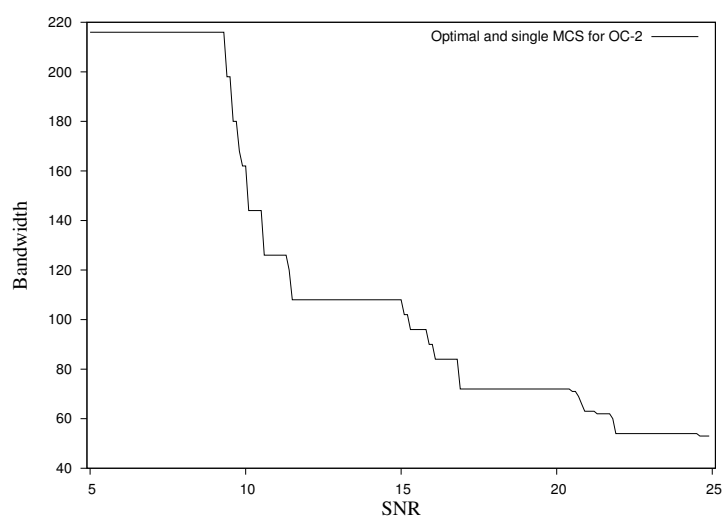

Fig. 9. The bandwidth for transmitting a data block vs. the SNR experienced by the designated receiver for OC-2

In Figure 9 we see that as the SNR value of the designated receiver increases, the bandwidth remains constant up to a certain point and then drops until it reaches a new step. This discrete drop between two constant bandwidth points can be explained as follows. Although we see an increase in the SNR, a new MCS is relevant only when the probability to receive a packet using this MCS becomes sufficiently high. As the SNR further increases, it becomes sufficiently high for transmitting fewer packets using this MCS. If we can transmit $K$ packets using this MCS, the bandwidth remains constant until the next MCS becomes relevant.

To simulate our OC-3 algorithms, we used $K=6$ and two groups of receivers. The group with the lower SNRs is assigned a probability threshold of 0.95 and the group with the higher SNRs is assigned a probability threshold of 0.99 . Figure 10 shows the bandwidth vs. the SNR experienced by the designated receiver in the group with the lower SNRs. The SNR value of the designated receiver in the group with the higher SNRs was set to be higher by $2.0 \mathrm{~dB}$. We saw, again, no differences between the optimal algorithm and the best MCS algorithm. Thus, only one curve is shown in the graph. Similar results were obtained when we increased the value of $K$ to 10 .

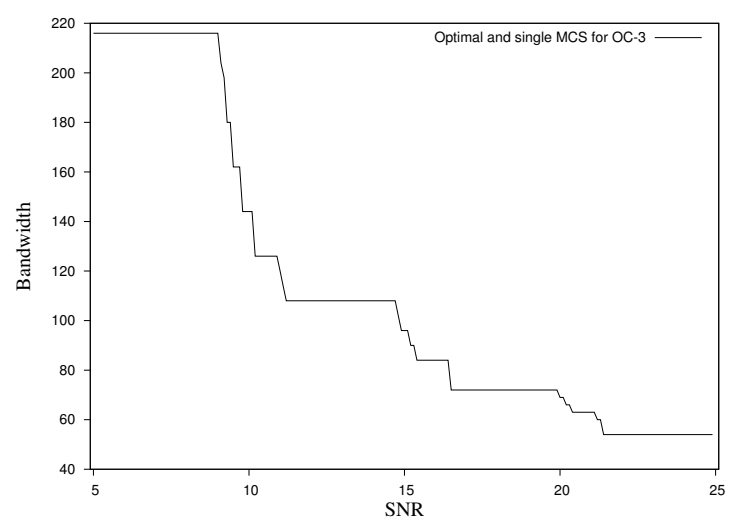

Fig. 10. The bandwidth for transmitting a data block vs. the SNR experienced by the designated receiver from the lower-quality subgroup for OC-3

When we used 3 OC-3 subgroups with probability thresholds of $0.95,0.99$ and 0.999 and a $2.0 \mathrm{~dB}$ difference between the SNRs of the designated receivers, we got very similar results.

For both OC-2 and OC-3, we found very specific scenarios for which the optimal algorithm reduces the bandwidth consumed by the single MCS algorithm. Since the maximum improvement we found was smaller than $8 \%$, and since in most scenarios no difference was found, we believe that the polynomial time single MCS algorithm should be preferred for OC-2 and OC-3, unless the number $N$ of MCSs is very small (2-3).

\section{CONCLUSIONS}

We defined a new problem, called RM-AMC, that arises when a base station in a broadband wireless network wishes to multicast information to a large group of nodes and to guarantee some level of reliability using Application layer FEC codes with or without ARQ. The problem is to determine which PHY layer MCS the base station should use for each packet. RMAMC was shown to have several variants, depending on the 
number of transmission rounds the sender can use. We defined an optimization criterion, referred to as $\mathrm{OC}-1$. We showed that RM-AMC(OC-1) is NP-hard for any fixed number of rounds. We then presented several algorithms for one or more rounds and studied their performance under different conditions. We then considered two other optimization criteria, referred to as OC-2 and OC-3. We showed that RM-AMC remains NPhard, and compared the performance of the optimal and the single MCS algorithms for OC-2 and OC-3 under different conditions.

\section{REFERENCES}

[1] 3GPP. Introduction of the Multimedia Broadcast/Multicast Service(MBMS) in the Radio Access Network (RAN), Mar. 2006

[2] B. Adamson, C. Bormann, M. Handley, and J. Macker. Negativeacknowledgment (NACK) - oriented reliable multicast (NORM) building blocks. RFC-3941, Nov. 2004.

[3] B. Adamson, C. Bormann, M. Handley, and J. Macker. Negativeacknowledgment (NACK) - oriented reliable multicast (NORM) protocol. RFC-3940, Nov. 2004.

[4] K. Balachandran, D. Calin, F. C. Cheng, N. Joshi, J. H. Kang, A. Kogiantis, K. Rausch, A. N. Rudrapatna, J. P. Seymour, and J. Sun. Design and analysis of an IEEE 802.16e-based OFDMA communication system. Bell Labs Technical Journal, 11(4):53-73, 2007.

[5] C. R. Berger, S. Zhou, Y. Wen, P. Willett, and K. Pattipati. Optimizing joint erasure- and error-correction coding for wireless packet transmissions. IEEE Transactions on Wireless Communications, 7(11):45864595, Nov. 2008.

[6] T. H. Cormen, C. E. Leiserson, and R. L. Rivest. Introduction to Algorithms. The MIT Press, 1990.

[7] S. Fashandi, S. Oveisgharan, and A. Khandani. Coding over an erasure channel with a large alphabet size. IEEE International Symposium on Information Theory, pages 1053-1057, July 2008.

[8] G.D.Papadopoulos, G.Koltsidas, and F.N.Pavlidou. Two hybrid ARQ algorithms for reliable multicast communications in UMTS networks. IEEE Communications Letters, 10(4):260-262, 2006.

[9] C. S. Hwang and Y. Kim. An adaptive modulation method for multicast communications of hierarchical data in wireless networks. In ICC, pages 896-900, 2002.

[10] Institute of Electrical and Electronics Engineers Inc. IEEE Draft Standard for Local and Metropolitan Area Networks - Part 16: Air Interface for Broadband Wireless Access Systems, June 2008

[11] K. Kar, S. Sarkar, and L. Tassiulas. Optimization based rate control for multirate multicast sessions. In INFOCOM, pages 123-132, 2001.

[12] H. Kellerer, U. Pferschy, and D. Pisinger. Knapsack Problems. Springer, 2004.

[13] J. Kim and D. H. Cho. Enhanced adaptive modulation and coding schemes based on multiple channel reportings for wireless multicast systems. In Vehicular Technology Conference, pages 725-729, 2005.

[14] J. Kim, J. Cho, and H. Shin. Resource allocation for scalable video broadcast in wireless cellular networks. In WiMob, volume 2, pages 174-180, Aug. 2005.

[15] H. Lenstra. Integer programming with a fixed number of variables. Math. Oper. Res., 8:538-548, 1983.

[16] D. Li and D. R. Cheriton. Evaluating the utility of FEC with reliable multicast. In ICNP, pages 97-105, 1999.

[17] M. Luby, L. Vicisano, J. Gemmell, L. Rizzo, M. Handley, and J. Crowcroft. The use of Forward Error Correction (FEC) in reliable multicast. RFC-3453, Dec. 2002.

[18] F. J. MacWilliams and N. J. A. Sloane. The theory of Error-Correcting Codes. Amsterdam, The Netherlands, 1977.

[19] J. Nonnenmacher, E. Biersack, and D. F. Towsley. Parity-based loss recovery for reliable multicast transmission. IEEE/ACM Trans. Netw. 6(4):349-361, 1998.

[20] M. Pursley and J. Shea. Multimedia multicast wireless communications with phase-shift-key modulation and convolutional coding. IEEE Journal on Selected Areas in Communications, 17(11):1999-2010, Nov. 1999.

[21] F. Retnasothie, M. Ozdemir, T. Yucek, H. Celebi, J. Zhang, and R. Muththaiah. Wireless IPTV over WiMAX: Challenges and applications. In WAMICON, pages 1-5, Dec. 2006.
[22] L. Rizzo and L. Vicisano. RMDP: An FEC-based reliable multicast protocol for wireless environments. ACM SIGMOBILE Mobile Computing and Communications Review, 2(2), Apr. 1998.

[23] D. Rubenstein, J. F. Kurose, and D. F. Towsley. A study of proactive hybrid FEC/ARQ and scalable feedback techniques for reliable, realtime multicast. Computer Communications, 24(5-6):563-574, 2001.

[24] J. She and P. H. Ho. Cooperative coded video multicast for IPTV services under EPON-WiMAX integration. IEEE Communications Magazine, pages 104-110, Aug. 2008.

[25] J. She, F. Hou, P. H. Ho, and L. L. Xie. IPTV over WiMAX: Key success factors, challenges, and solutions [advances in mobile multimedia]. IEEE Communications Magazine, 45(8):87-93, Aug. 2007.

[26] J. She, X. Yu, P. H. Ho, and E. H. Yang. A cross-layer design framework for robust IPTV services over IEEE 802.16 networks. IEEE Journal on Selected Areas in Communications, 27(2):235-245, Feb. 2009.

[27] Y. R. Yang, M. S. Kim, and S. S. Lam. Optimal partitioning of multicast receivers. In ICNP, pages 129-140, 2000.

\section{APPENDIX}

\section{A. The Proof of Theorem 1}

To prove that 1-round RM-AMC $(\mathrm{OC}-1)$ is NP-hard, we present the Unbounded Subset Sum Problem (USSP) [12]. The instance of USSP is a set $S$ of item types $s_{1}, s_{2}, \ldots, s_{m}$ and a capacity $C$. Each type $s_{i}$ has a weight $w\left(s_{i}\right)$. The objective is to find a vector $S^{\prime}=\left(s_{1}^{\prime}, \ldots, s_{m}^{\prime}\right)$ of items whose aggregated weight $\sum_{i=1}^{m} s_{i}^{\prime} \cdot w\left(s_{i}\right)$ is maximum but not larger than $C$.

USSP is known to be NP-hard in the weak sense ${ }^{2}$ [12]. We reduce the decision version of USSP into the decision version of 1-round RM-AMC(OC-1). Recall that in a decision version of a problem, an algorithm is only expected to tell whether or not a solution with a specified value exists. In the decision version of 1-round RM-AMC(OC-1), the algorithm only needs to tell whether there is a transmission configuration whose total bandwidth cost is $B$ and the probability that the designated receiver will correctly decode the data block is $P$.

Given an input for USSP, we translate it into an input for 1-round RM-AMC(OC-1) in the following way. Every item type $s_{i} \in S$ is transformed into a function $f_{i}(S N R)=1-$ $2^{-w\left(s_{i}\right) / C}$ that determines the probability to correctly receive an MCS- $i$ packet for a given SNR value. The size of a packet encoded using MCS- $i$ is equal to $w\left(s_{i}\right)$. Note that with this transformation we have $N=|S|=m$ MCSs. In addition, for the RM-AMC(OC-1) instance, we set $P \leftarrow \frac{1}{2}, K \leftarrow 1$ and $B \leftarrow C$

We now show that there exists an USSP solution that uses the entire capacity $C$ if and only if there exists a transmission configuration with bandwidth $B=C$ such that the probability that the designated receiver will correctly decode the data block is $P=\frac{1}{2}$. Let $\tau_{i}$ be the number of packets transmitted using MCS- $i$ in the solution for RM-AMC(OC-1). The probability that the designated receiver will not receive the data block correctly is equal to the probability that it will not receive any packet, namely, $\prod_{j=1}^{N}\left(1-f_{j}(S N R)\right)^{\tau_{j}}=$ $\prod_{j=1}^{N} 2^{-w\left(s_{j}\right) \cdot \tau_{j} / C}=2^{-\sum_{j=1}^{N} w\left(s_{j}\right) \cdot \tau_{j} / C}$. If there is a solution for USSP that uses the entire capacity $C$, then there is a transmission configuration that uses the entire bandwidth $B=C$. In such a case, $\sum_{j=1}^{N} w\left(s_{j}\right) \cdot \tau_{j}=C$ and the probability that the designated receiver will correctly decode

\footnotetext{
${ }^{2} \mathrm{~A}$ problem is NP-hard in the weak sense if it is NP-hard when the input is represented as a binary string.
} 
the data block is $\frac{1}{2}$. If a solution for USSP does not exist, then for every transmission configuration, $\sum_{j=1}^{N} w\left(s_{j}\right) \cdot \tau_{j}<C$, and the probability that the designated receiver will correctly decode the data block is $<\frac{1}{2}$.

\section{B. The Proof of Theorem 2}

In Theorem 1, we proved that 1-round RM-AMC(OC-1) is NP-hard even if $K=1$. We now reduce the instance of the 1-round $\mathrm{RM}-\mathrm{AMC}(\mathrm{OC}-1)$ decision problem considered in Theorem 1 to an instance of the $R$-round RM-AMC(OC-1) decision problem for a constant $R>1$. The reduction is trivial: the input remains the same and the decision to be made by an $R$-round RM-AMC(OC-1) algorithm is whether there exists an $R$-round algorithm with total bandwidth $\operatorname{cost} B$ for which the probability that the designated receiver will correctly decode the data block is $P$.

If there is a solution for the considered 1-round RM$\mathrm{AMC}(\mathrm{OC}-1)$ instance, the same transmission configuration can be used in the first round of the $R$-round RM-AMC(OC-1) problem, and in the remaining $R-1$ rounds no packet is sent. If there is a solution for the reduced $R$-round RM-AMC(OC-1) problem, then, since $K=1$, there is an algorithm composed of $R$ transmission configurations where the $i$ th transmission configuration is used in the $i$ th round. The event that occurs if in the $i$ th round the receiver correctly receives at least 1 packet is denoted $D_{i}$. Note that since $K=1$, this is equivalent to the event that the receiver correctly decodes the data block after the $i$ th round. The probability that the receiver will correctly decode the data block is $\operatorname{Pr}\left(D_{1} \vee D_{2} \vee \cdots \vee D_{R}\right)=$ $1-\operatorname{Pr}\left(D_{1}^{c} \wedge D_{2}^{c} \wedge \cdots \wedge D_{R}^{c}\right)$, where $D_{i}^{c}$ is the complement of $D_{i}$. Let $\overline{\tau(i)}$ be the transmission configuration used in the $i$ th round and let $\overline{\tilde{\tau}}=\sum_{i=1}^{R} \overline{\tau(i)}$. The probability for correctly decoding the data block when using $\overline{\tilde{\tau}}$ in the considered 1-round RM$\mathrm{AMC}(\mathrm{OC}-1)$ instance is exactly $1-\operatorname{Pr}\left(D_{1}^{c} \wedge D_{2}^{c} \wedge \cdots \wedge D_{R}^{c}\right)$. The bandwidth of $\overline{\tilde{\tau}}$ is equal to the bandwidth used in the solution for the reduced $R$-round RM-AMC(OC-1) problem. Hence, there is a solution for the considered 1-round RM$\mathrm{AMC}(\mathrm{OC}-1)$ instance and the reduction holds.

\section{The Proof of Theorem 3}

We prove the theorem by induction on the number of rounds $r$. For $r=1$, the correctness of Eq. 2 is straightforward, because we select the maximum value of $G^{\bar{\tau}}[\geq k]$ over all transmission configurations with bandwidth $b$. By the induction hypothesis, Eq. 2 calculates $H(k, b, r)$ correctly for every $r<l$ for a given $l>1$ and for every $k, b$. We now show that Eq. 2 also calculates $H(k, b, l)$ correctly. By the induction hypothesis, for every $i G^{\bar{\tau}}[i] \cdot H(\max (k-i, 0), b-c, l-1)$ is equal to the maximum probability that the designated receiver will correctly receive at least $k$ packets using $l$ rounds and bandwidth $b$, assuming that in round $l$ the transmission configuration $\bar{\tau}$ is used and exactly $i$ packets are correctly received during this round. Since the summation in Eq. 2 is performed for $0 \leq i \leq c$, it is equal to the probability that the designated receiver will correctly receive at least $k$ packets using $l$ rounds and bandwidth $b$ assuming that in round $l$ the transmission configuration $\bar{\tau}$ is used. Since the selected summation value is the maximum over all transmission configurations with bandwidth $\leq b$, the theorem holds.

\section{The Proof of Theorem 4}

We prove the theorem by induction on the number of rounds $r$. For $r=1$, the correctness of Eq. 5 is straightforward, because we select the maximum between the performance of Alg. 2 and that of a single MCS (for every MCS- $j$ ) in the single round. Assume that Eq. 5 calculates $M(k, b, r)$ correctly for every $r<l$ for a given $l>1$ and for every $k, b$. We now show that Eq. 5 also calculates $M(k, b, l)$ correctly. By the induction hypothesis, $\sum_{i=0}^{c} U_{c}[i] \cdot M(\max (k-i, 0), b-c, l-1)$ is equal to the maximum probability that the designated receiver will correctly receive at least $k$ packets using $l$ rounds and bandwidth $b$, while Alg. 2 is used with bandwidth $c$ in the $l$ th round. Now, note that $\sum_{i=0}^{c} S_{c}^{j}[i] \cdot M(\max (k-i, 0), b-c, l-1)$ is equal to the maximum probability that the designated receiver will correctly receive at least $k$ packets using $l$ rounds and bandwidth $b$ where in round $l$ only MCS- $j$ packets are transmitted and the total bandwidth of this round is $c$. Since in Eq. 5 we consider every $0 \leq c \leq b$ and every $1 \leq j \leq N$ and select the maximal value, the theorem holds.

\section{E. The Proof of Theorem 5}

We prove the theorem by induction on the bandwidth $b$. For $b=0$, the correctness of Eq. 6 is straightforward because no packet can be transmitted. By the induction hypothesis we assume that Eq. 6 calculates $F(k, b)$ correctly for every $b<$ $l$ where $l>0$ and for every $k$. We now show that Eq. 6 calculates $F(k, l)$ correctly. First, note that by Observation 3 it is sufficient to consider the cases where in every round a single packet is transmitted. We first consider the case where for every $j, b_{j}>l$. Although the bandwidth is $>0$, in this case we still cannot transmit any packet. Thus, $F(k, l)=F(k, 0)$, and Eq. 6 holds. If $b_{j} \geq l$ holds for some $j$, the expression $p^{j}$. $F\left(k-1, l-b_{j}\right)+\left(1-p^{j}\right) \cdot F\left(k, l-b_{j}\right)$ is equal to the maximum probability that the designated receiver will correctly receive at least $k$ packets using bandwidth $l$, where in round $l$ an MCS- $j$ packet is transmitted. Since we select the maximum value over all values of $j$ from which a packet can be sent using MCS- $j\left(b_{j}<b\right)$, Eq. 6 calculates $F(k, l)$ correctly in this case as well.

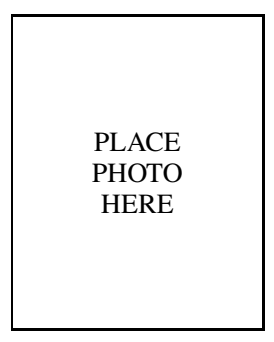

Reuven Cohen (M'93, SM'99) received his B.Sc., M.Sc. and Ph.D. degrees in Computer Science from the Technion - Israel Institute of Technology, completing his Ph.D. studies in 1991. From 1991 to 1993, he was with the IBM T.J. Watson Research Center, working on protocols for high speed networks. Since 1993, he has been a professor in the Department of Computer Science at the Technion. He has also been a consultant for numerous companies, mainly in the context of protocols and architectures for broadband access networks. Dr. Cohen has served as an editor of the IEEE/ACM Transactions on Networking and the ACM/Kluwer Journal on Wireless Networks (WINET). He is the technical program co-chair of Infocom'2010. Dr. Cohen is a senior member of the IEEE and heads the Israeli chapter of the IEEE Communications Society. 


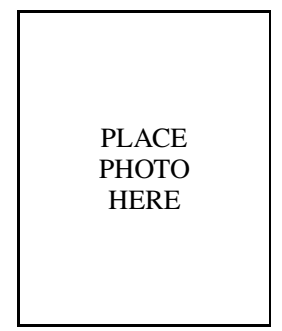

Guy Grebla received his B.A. degree in computer science from the Technion - Israel Institute of Technology, Haifa, Israel, in 2003. He is now pursuing an M.Sc. in the field of wireless networking.

\begin{tabular}{|c|}
\hline \\
\\
PLACE \\
PHOTO \\
HERE \\
\end{tabular}

Liran Katzir received his B.A., M.A., and Ph.D. degrees in computer science from the Technion Israel Institute of Technology, Haifa, Israel, in 2001, 2005 , and 2008 respectively. His $\mathrm{PhD}$. thesis is about scheduling in advanced wireless networks.

Dr. Katzir is now a member of the networking research group in the Technion's CS department, working on technologies for the fourth generation cellular network. 